\title{
Case Report \\ Prosthetic Aortic Valve Stenosis in End-Stage Renal Failure
}

\author{
Michael Mao, ${ }^{1}$ Mirelle El Ters, ${ }^{1}$ Sunil Mankad, ${ }^{2}$ Mira Keddis, ${ }^{1}$ \\ Soon Park, ${ }^{3}$ and Qi Qian ${ }^{1}$ \\ ${ }^{1}$ Division of Nephrology and Hypertension, Department of Medicine, Mayo Clinic, Rochester, MN 55902, USA \\ ${ }^{2}$ Division of Cardiology, Department of Medicine, Mayo Clinic, Rochester, MN 55902, USA \\ ${ }^{3}$ Department of Surgery, Mayo Clinic, Rochester, MN 55902, USA
}

Correspondence should be addressed to Qi Qian, qian.qi@mayo.edu

Received 2 February 2011; Accepted 18 March 2011

Academic Editor: Greg Tesch

Copyright () 2011 Michael Mao et al. This is an open access article distributed under the Creative Commons Attribution License, which permits unrestricted use, distribution, and reproduction in any medium, provided the original work is properly cited.

\begin{abstract}
Although renal failure is one of the known comorbidities associated with rapid progression of aortic stenosis, it is unclear whether hemodialysis alters the progression of prosthetic aortic valve stenosis. We describe a 79-year-old female who underwent bioprosthetic aortic valve replacement 8 years ago with stable prosthetic valve area for the initial 6 years. In the last two years, coinciding with the initiation of maintenance hemodialysis, she developed progressive prosthetic valve stenosis to the point of clinical decompensation. She underwent a second prosthetic aortic valve replacement with symptom resolution. This case suggests that circulating milieu in end-stage renal failure and dialysis can accelerate the progression of prosthetic aortic valve stenosis. More frequent clinical followup and surveillance echocardiogram for dialysis patients with bioprosthetic aortic valve may facilitate timely management of valvular stenosis.
\end{abstract}

\section{Introduction}

The natural history of aortic stenosis can be affected by a number of comorbidities. Although renal failure is one of the known comorbidities for rapid progression of aortic stenosis, it is unclear whether hemodialysis alters the development and progression of prosthetic aortic valve stenosis. We describe a patient with a bioprosthetic aortic valve who acquired accelerated aortic stenosis following the initiation of hemodialysis for end-stage renal failure.

\section{Case Report}

A 79-year-old female was admitted for dyspnea with hypoxia in October 2010. She had longstanding history of hypertension, hyperlipidemia, and chronic kidney dysfunction. In 2002, she underwent aortic valve replacement with a $23 \mathrm{~mm}$ Carpentier-Edward tissue aortic valve for critical aortic stenosis related to bicuspid aortic valve. In 2008, she was initiated on hemodialysis for end-stage renal failure secondary to biopsy-proven hypertensive nephrosclerosis. She received dialysis via a right internal jugular tunneled dialysis catheter.
Arterial-venous fistula was not created per her request. She was on a stable medical regimen of metoprolol, gemfibrozil, ezetimibe, allopurinol, and aspirin. For her end-stage renal failure, she was maintained on calcium acetate $1334 \mathrm{mg}$ with each meal as well as paricalcitol $7.5 \mathrm{mcg}$ and darbepoetin with hemodialysis. Since February of 2010, she began to experience progressive worsening dyspnea with notable orthopnea, paroxysmal nocturnal dyspnea, and decreasing exercise tolerance. Transthoracic echocardiograms (see Section 3) revealed progressive worsening prosthetic valve stenosis.

Physical examination on admission showed blood pressure $94 / 54 \mathrm{mmHg}$, respiratory rate $30 /$ minute, oxygen saturation $93 \%$ on $70 \%$ oxygen via a facemask, pulse $86 /$ minute, and diffuse crackles in bilateral lung fields. She had diminished bilateral carotid upstroke and a 4/6 systolic ejection murmur across the precordium associated with soft aortic component of the second heart sound. There was no lower extremity edema. Laboratory results included hemoglobin $11 \mathrm{~g} / \mathrm{dL}$, leukocyte count $10.6 \times 10^{9} / \mathrm{L}$, sodium $141 \mathrm{mmol} / \mathrm{L}$, potassium $5.5 \mathrm{mmol} / \mathrm{L}$, bicarbonate $21 \mathrm{mmol} / \mathrm{L}$, and creatinine $5.4 \mathrm{mg} / \mathrm{dL}$. A chest X-ray showed florid pulmonary 
edema. She was provisionally diagnosed with fluid overload and heart failure.

\section{Imaging Studies}

Transthoracic echocardiogram was performed, which showed severe prosthetic aortic valve stenosis. On review of prior echocardiograms, there was a rapid progression of the valve stenosis along with elevated serum calcium-phosphate product levels following the initiation of hemodialysis (Figure 1 and Table 1).

\section{Clinical Followup}

A coronary angiogram was performed which showed an approximately 30\% obstruction of left anterior descending artery in addition to the aortic stenosis. She subsequently underwent a second aortic valve replacement with a $23 \mathrm{~mm}$ Mitroflow bioprosthetic valve and coronary artery bypass surgery with left internal mammary artery graft to the left anterior descending artery. Prior to the operation, an option of mechanical valve versus tissue valve was discussed. A decision to proceed with another tissue valve was made on the basis of her age, risks associated with chronic anticoagulation, and her preference. Intraoperatively, her bioprosthetic aortic valve was visibly noted to be heavily calcified, and the cusps were retracted with small vegetations attached to them. There was no cusp tear, thrombus, or fibrous pannus ingrowth. Following the surgery, her systemic blood pressure improved (110-130/70-80 $\mathrm{mmHg}$ ) and respiratory symptoms resolved. She was discharged from the hospital, and remained asymptomatic at one-month followup.

\section{Discussion}

We present a patient who developed accelerated stenosis in the bioprosthetic aortic valve coincident with the initiation of hemodialysis, suggesting that the circulating milieu in end-stage renal failure and dialysis can adversely affect the progression of prosthetic aortic valve stenosis.

Aortic valve stenosis, defined as a reduction in the aortic valve area (normal range $2-4 \mathrm{~cm}^{2}$ ) leading to an increase in transvalvular jet velocity $(>2.6 \mathrm{~m} / \mathrm{s})$ and decrease in cardiac output, is the most common valvular abnormality in both the general population and patients with renal failure. It is the most frequent cause of death associated with valvular heart disease and an independent risk factor for death in dialysis patients [1-4]. Aortic stenosis can be caused, alone or in combination, by congenital bicuspid valve or acquired valvular degenerative calcification related to longstanding hypertension, hyperlipidemia, calcific disease, and chronic inflammatory conditions such as rheumatic disease [5]. In recent decades, with increases in life expectancy, the major cause of aortic stenosis has shifted from congenital valvular abnormality, that is, bicuspid aortic valve, to acquired degenerative valvular calcification $[1,6,7]$.
As aortic stenosis is known to progress, regular monitoring to facilitate timely treatment before irreversible myocardial damage is lifesaving $[1,2]$. In practice, progression of aortic stenosis is routinely monitored by clinical observation and by echocardiographic examination, which determines the aortic valve area, mean transvalvular pressure gradient, and maximal jet velocity. The current practice guidelines for monitoring aortic stenosis are to perform periodic transthoracic echocardiography, every 3 to 5 years for mild aortic stenosis (defined as a valve area of $1.2-1.5 \mathrm{~cm}^{2}$, mean gradient of $<25 \mathrm{mmHg}$, or jet velocity of $2.5-3.0 \mathrm{~m} / \mathrm{s}$ ), 1 to 2 years for moderate stenosis (valve area of $0.7-1.2 \mathrm{~cm}^{2}$, mean gradient of $25-40 \mathrm{mmHg}$, or jet velocity of $3.0-4.0 \mathrm{~m} / \mathrm{s}$ ), and yearly for severe stenosis (valve area of $<0.7 \mathrm{~cm}^{2}$, mean gradient of $>40 \mathrm{mmHg}$, or jet velocity of $>4.0 \mathrm{~m} / \mathrm{s}$ ) $[8,9]$. On average, aortic valve area decreases by $0.05-0.1 \mathrm{~cm}^{2} /$ year, jet velocity increases by approximately $0.3 \mathrm{~m} / \mathrm{s} / \mathrm{year}$, and mean pressure gradient increases by approximately $7 \mathrm{mmHg} /$ year [8].

Hemodialysis has been shown to correlate with an increased prevalence $(40-52 \%)$ and a rapid progression of native aortic valve stenosis $[1,3,8,10-12]$. In a retrospective study of 110 patients on maintenance hemodialysis, Ureña et al. found an increased incidence of aortic stenosis and an accelerated reduction of aortic valve area from 1.24 to $0.66 \mathrm{~cm}^{2} / \mathrm{m}^{2}$ of body surface area in $16.8 \pm 1.9$ months [3]. Such changes correspond to an average reduction in the valve area of $0.23 \mathrm{~cm}^{2} /$ year, a progression rate over two times that of the general population $\left(0.05-0.1 \mathrm{~cm}^{2} /\right.$ year $)[3,8,13]$. Perkovic et al. provided further support in a retrospective case-control study [14]. They compared progression of aortic stenosis in dialysis patients to sex-matched nondialysis individuals, 28 and 56 patients in each group, respectively. They found an average decrease in the aortic valve area of $0.19 \mathrm{~cm}^{2} /$ year and increase in the peak transvalvular gradient of $4.9 \mathrm{mmHg} /$ year in the dialysis patients, a significantly rapid progression compared to the nondialysis patients, $0.07 \mathrm{~cm}^{2} /$ year and $2.5 \mathrm{mmHg} /$ year, respectively. Recently, a study by Zentner et al. showed that when matched for aortic stenosis severity at the study entry, dialysis patients, compared to nondialysis patients, had a decreased event-free survival (event defined as death or aortic valve replacement), once again correlating to accelerated aortic stenosis in dialysis patients [15].

The pathophysiology underlying the rapid progression of aortic stenosis in dialysis patients has not been fully elucidated. A reduced aortic valve area at baseline, preexisting valvular calcification, and high levels of circulating calciumphosphate product and parathyroid hormone have all been proposed to contribute to the progression of stenosis $[3,16-$ 19]. Data on progression of prosthetic aortic valve stenosis in renal failure patients are scarce. Only two case reports were found in the literature. Bradley et al. described a hemodialysis patient who received a bioprosthetic (heterograft) aortic valve for native bacterial valve endocarditis. About 15 months later, the patient developed symptoms of aortic stenosis. Echocardiography showed a critical prosthetic valve stenosis with heavy valvular calcification. The patient underwent a second tissue valve (Bjork-Shiley prosthesis) 

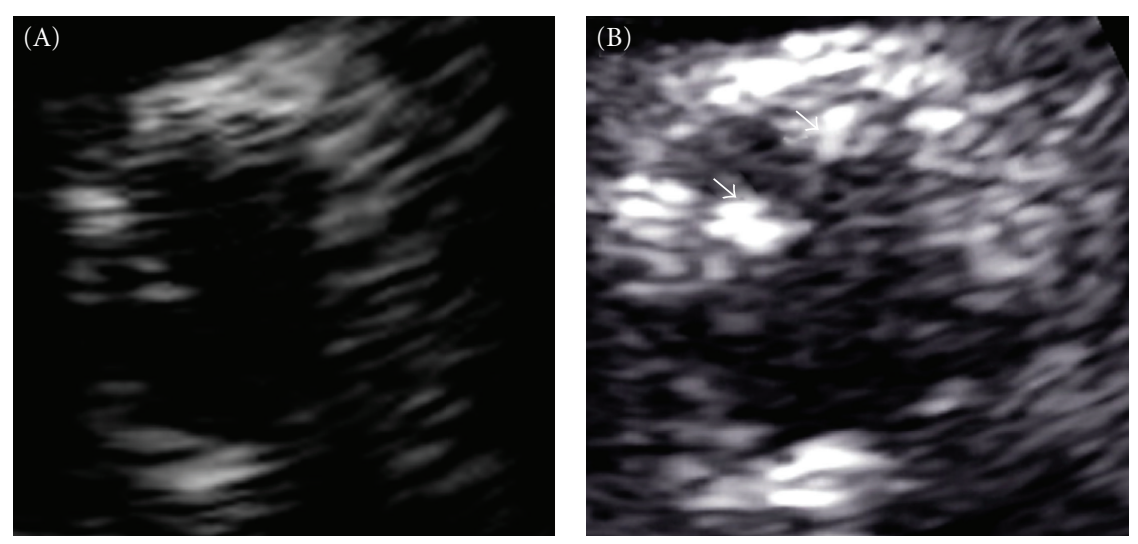

(a)
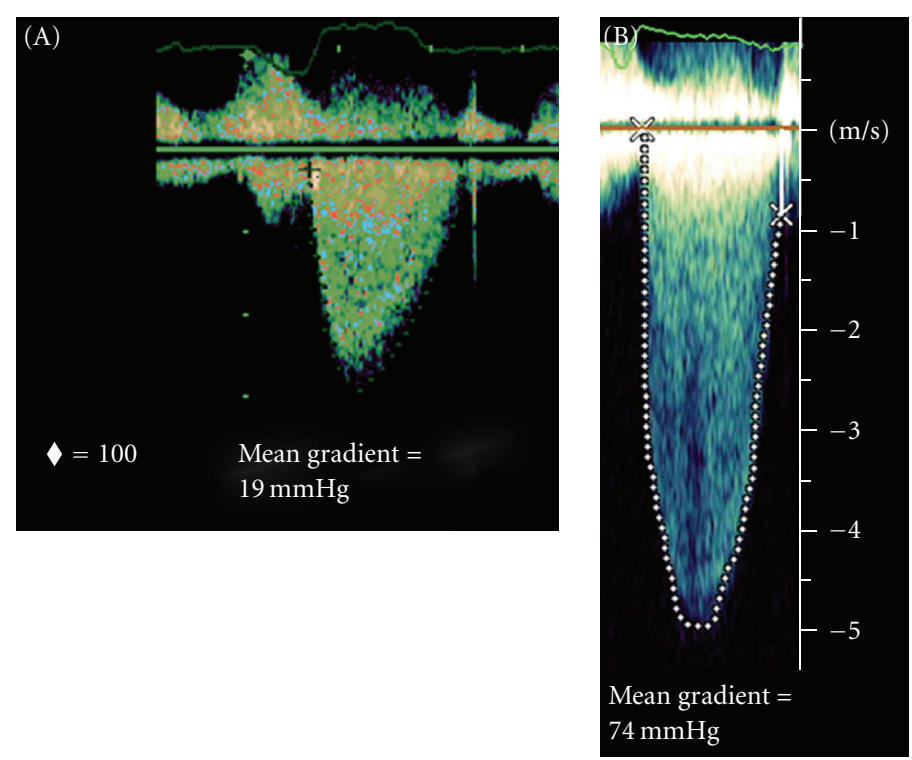

(b)
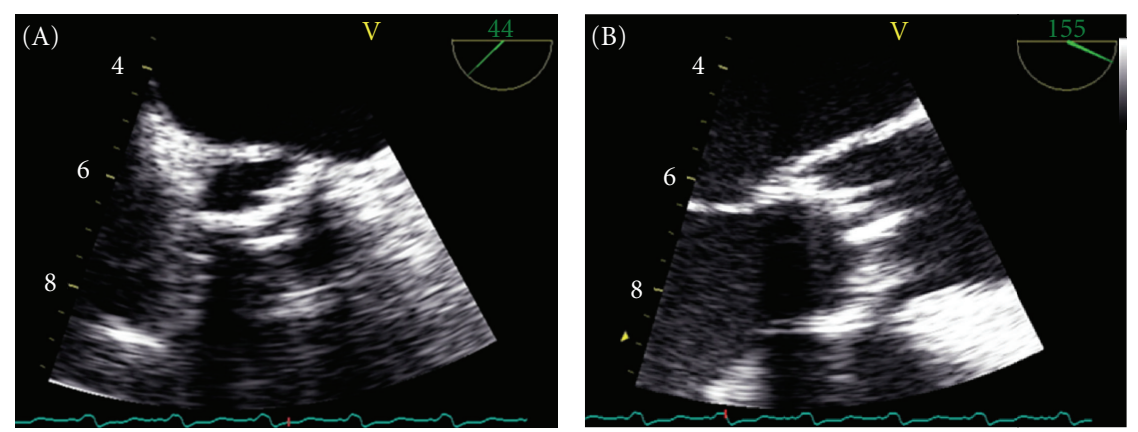

(c)

FIGURE 1: (a) shows short-axis images of the aortic valve prosthesis. (A) is the aortic valve prosthesis 5 years after implantation demonstrating minimal calcification and thin, barely visible, leaflets. Shown on (B) is the prosthetic aortic valve about 2-years after beginning dialysis demonstrating heavy leaflet calcification (arrows). (b) shows the aortic valve prosthetic mean gradient by spectral continuous wave Doppler echocardiography demonstrating a normal gradient 5 years after implantation (A) and a markedly increased gradient shortly after beginning dialysis (B), corresponding to the images in (a). (c) shows the intraoperative transesophageal echocardiographic images of the stenotic aortic valve prosthesis at the time of the second aortic valve operation. Short-axis image on the left (A) and long-axis image on the right (B) demonstrate marked leaflet calcification, thickening, and severe stenosis. 
TABLE 1: Summary data of serial echocardiography and serum calcium and phosphate concentration.

\begin{tabular}{|c|c|c|c|c|}
\hline \multirow{2}{*}{ Test Date } & \multicolumn{3}{|c|}{ Transthoracic echocardiogram } & \multirow{2}{*}{$\begin{array}{l}\text { Calcium-phosphate } \\
\text { Product (calcium } \times \\
\text { phosphate })(\mathrm{mg} / \mathrm{dL})\end{array}$} \\
\hline & Aortic valve area $\left(\mathrm{cm}^{2}\right)$ & Transvalvular gradient (mmHg) & Velocity (m/sec) & \\
\hline Sept. 2002 & 0.73 & 56 & 4.7 & \\
\hline Oct. 2002 & \multicolumn{3}{|c|}{ 1st aortic valve replacement operation } & $35.7(9.4 \times 3.8)$ \\
\hline Oct. 2004 & 1.78 & 22 & 3.6 & \\
\hline Oct. 2006 & $*$ & 17 & 2.8 & \\
\hline July 2007 & 1.87 & 19 & 3.0 & \\
\hline April 2008 & 1.74 & 16 & 2.7 & \\
\hline May 2008 & \multicolumn{3}{|c|}{ Commencement of dialysis } & $64.8(7.9 \times 8.2)$ \\
\hline March 2010 & 0.88 & 40 & 4.3 & \\
\hline April 2010 & $*$ & 49 & 4.3 & $53.9(9.3 \times 5.8)$ \\
\hline Sept. 2010 & 0.73 & 74 & 5.1 & $51.9(9.1 \times 5.7)$ \\
\hline Oct. 2010 & \multicolumn{3}{|c|}{2 nd aortic valve replacement operation } & $47.2(8.9 \times 5.3)$ \\
\hline Nov. 2010 & 2.08 & 21 & & \\
\hline
\end{tabular}

*: Unavailable (missing data).

replacement that resulted in symptom resolution [20]. In another report by Hahn and Maze a hemodialysis patient who had a bioprosthetic aortic valve replacement for native calcific aortic stenosis developed symptoms of heart failure due to critical prosthetic valve stenosis only 20 months after the valve replacement surgery. The patient subsequently had a second St. Jude mechanical valve replacement with resolution of the symptoms [21]. The progression of prosthetic valve stenosis in our patient seemed to follow the pattern of these reported cases. Her advanced age, possible subclinical prosthetic valve infection (suggested by small vegetations on the resected valve), elevated calcium-phosphate product level, as shown in Table 1, and chronic dialysis could all be the potential factors contributing to the accelerated progression of bioprosthetic valve stenosis. We speculate that perhaps if the reversible factors, that is, calcium-phosphorous product and subclinical infection, were more tightly controlled, the rate of her valve degeneration might have been tempered.

Whether dialysis patients are more suited for having bioprosthetic or mechanical valve replacement remains unclear. Because calcific stenosis of prosthetic valve is known to occur almost exclusively with bioprosthetic valves, it was advocated that for dialysis patients mechanical valve might be a more appropriate choice. However, three retrospective studies have challenged this notion. Kaplon et al. conducted an initial study on 42 chronic hemodialysis patients who underwent mitral and aortic valve replacements; 17 received mechanical valves and 25 bioprosthesis. Patient survival at 3 and 5 years between two groups was similar [22]. Subsequently, Chan et al. studied 69 dialysis patients with valve replacement surgery; 47 had aortic valve replacement, 33 bioprosthetic and 14 mechanical valves. That founds the 5-year of freedom from valve related complications were similar for bioprosthetic and mechanical valves [23]. Bioprosthetic valves were more prone to calcification while mechanical valves had increased risks of valve related thrombosis, thromboembolism, and hemorrhage due to anticoagulation. It seems that the benefit of less prosthetic calcification with mechanical valves is offset by their thrombogenic and anticoagulation associate complications. Similarly, a recent study by Tanaka et al. found no significant difference in late outcomes (an average followup of $42 \pm 31$ months) between bioprosthetic $(n=22)$ and mechanical $(n=51)$ aortic valve replacement in dialysis patients [24]. Further study is needed to confirm these findings.

This case has important clinical implications. Chronic kidney disease population is increasing, so is the dialysis population. Aortic stenosis is the most common valvular abnormality among dialysis patients and is much more prevalent than in the general population. Although the occurrence of rapid prosthetic valve stenosis appears sporadic, with increasing number of dialysis patients, it is important to investigate the underlying risk factors for this phenomenon so that at-risk patients could be identified and preventive measures implemented. Moreover, as untreated aortic stenosis can be fatal, the recognition that prosthetic aortic valve may become rapidly stenotic in dialysis patients should increase alertness of this diagnostic possibility, leading to an appropriate investigation (echocardiography in this case) and timely treatment, thereby avoiding irreversible cardiac damage and death.

In summary, we present a patient with accelerated progression of prosthetic aortic valve stenosis following the initiation of dialysis. Echocardiography is essential in making the diagnosis. Although further study is needed to better understand the natural history of prosthetic valve stenosis in dialysis patients, this case suggests that, in dialysis patients with prosthetic aortic valve, the valve stenosis may recur at an accelerated pace. A tailored approach with more frequent clinical followup and surveillance echocardiogram may be necessary. 


\section{Acknowledgment}

The authors thank their patient for her permission to report this case.

\section{References}

[1] E. Umana, W. Ahmed, and M. A. Alpert, "Valvular and perivalvular abnormalities in end-stage renal disease," American Journal of the Medical Sciences, vol. 325, no. 4, pp. 237-242, 2003.

[2] A. Baglin, T. Hanslik, J. N. Vaillant, J. C. Boulard, L. Moulonguet-Doleris, and J. Prinseau, "Severe valvular heart disease in patients on chronic dialysis. A five-year multicenter French survey," Annales de Medecine Interne, vol. 148, no. 8, pp. 521526, 1997.

[3] P. Ureña, M. C. Malergue, B. Goldfarb, P. Prieur, C. GuédonRapoud, and M. Pétrover, "Evolutive aortic stenosis in hemodialysis patients: analysis of risk factors," Nephrologie, vol. 20, no. 4, pp. 217-225, 1999.

[4] C. M. Otto, B. K. Lind, D. W. Kitzman, B. J. Gersh, and D. S. Siscovick, "Association of aortic-valve sclerosis with cardiovascular mortality and morbidity in the elderly," The New England Journal of Medicine, vol. 341, no. 3, pp. 142-147, 1999.

[5] M. B. Lewin and C. M. Otto, "The bicuspid aortic valve: adverse outcomes from infancy to old age," Circulation, vol. 111, no. 7, pp. 832-834, 2005.

[6] C. M. Otto, J. Kuusisto, D. D. Reichenbach, A. M. Gown, and K. D. O'Brien, "Characterization of the early lesion of 'degenerative' valvular aortic stenosis: histological and immunohistochemical studies," Circulation, vol. 90, no. 2, pp. 844-853, 1994.

[7] C. S. Passik, D. M. Ackermann, J. R. Pluth, and W. D. Edwards, "Temporal changes in the causes of aortic stenosis: a surgical pathologic study of 646 cases," Mayo Clinic Proceedings, vol. 62, no. 2, pp. 119-123, 1987.

[8] R. O. Bonow, B. A. Carabello, K. Chatterjee et al., "2008 focused update incorporated into the ACC/AHA 2006 guidelines for the management of patients with valvular heart disease: a report of the American College of Cardiology/ American Heart Association Task Force on practice guidelines (writing committee to revise the 1998 guidelines for the management of patients with valvular heart disease): endorsed by the society of cardiovascular anesthesiologists, society for cardiovascular angiography and interventions, and society of thoracic surgeons," Circulation, vol. 118, no. 15, pp. e523e661, 2008.

[9] "Valvular stenosis," in Clinical Manual and Review of Transesophageal Echocardiography, J. P. Mathew and C. M. Ayoub, Eds., pp. 74-90, McGraw-Hill Medical, New York, NY, USA, 2005.

[10] E. R. Maher, M. Pazianas, and J. R. Curtis, "Calcific aortic stenosis: a complication of chronic uraemia," Nephron, vol. 47, no. 2, pp. 119-122, 1987.

[11] G. M. Novaro, R. Sachar, G. L. Pearce, D. L. Sprecher, and B. P. Griffin, "Association between apolipoprotein E alleles and calcific valvular heart disease," Circulation, vol. 108, no. 15, pp. 1804-1808, 2003.

[12] J. R. Ortlepp, F. Schmitz, V. Mevissen et al., "The amount of calcium-deficient hexagonal hydroxyapatite in aortic valves is influenced by gender and associated with genetic polymorphisms in patients with severe calcific aortic stenosis," European Heart Journal, vol. 25, no. 6, pp. 514-522, 2004.
[13] P. Faggiano, G. P. Aurigemma, C. Rusconi, and W. H. Gaasch, "Progression of valvular aortic stenosis in adults: literature review and clinical implications," American Heart Journal, vol. 132, no. 2, pp. 408-417, 1996.

[14] V. Perkovic, D. Hunt, S. V. Griffin, M. du Plessis, and G. J. Becker, "Accelerated progression of calcific aortic stenosis in dialysis patients," Nephron Clinical Practice, vol. 94, no. 2, pp. c40-45, 2003.

[15] D. Zentner, D. Hunt, W. Chan, F. Barzi, L. Grigg, and V. Perkovic, "Prospective evaluation of aortic stenosis in endstage kidney disease: a more fulminant process?" Nephrol Dial Transplant, vol. 26, no. 5, pp. 1651-1655, 2011.

[16] S. Kajbaf, J. P. Veinot, A. Ha, and D. Zimmerman, "Comparison of surgically removed cardiac valves of patients with ESRD with those of the general population," American Journal of Kidney Diseases, vol. 46, no. 1, pp. 86-93, 2005.

[17] E. R. Maher, G. Young, B. Smyth-Walsh, S. Pugh, and J. R. Curtis, "Aortic and mitral valve calcification in patients with end-stage renal disease," The Lancet, vol. 2, no. 8564, pp. 875877, 1987.

[18] T. Kume, T. Kawamoto, T. Akasaka et al., "Rate of progression of valvular aortic stenosis in patients undergoing dialysis," Journal of the American Society of Echocardiography, vol. 19, no. 7, pp. 914-918, 2006.

[19] S. Ribeiro, A. Ramos, A. Brandão et al., "Cardiac valve calcification in haemodialysis patients: role of calcium-phosphate metabolism," Nephrology Dialysis Transplantation, vol. 13, no. 8, pp. 2037-2040, 1998.

[20] J. R. Bradley, P. F. Williams, and D. B. Evans, "Aortic valve replacement in chronic renal failure," The Lancet, vol. 2, no. 8468, p. 1370, 1985.

[21] H. S. Hahn and S. S. Maze, "Rapid bioprosthetic valve degeneration in a patient undergoing hemodialysis," Annals of Internal Medicine, vol. 132, no. 10, p. 847, 2000.

[22] R. J. Kaplon, D. M. Cosgrove III, A. M. Gillinov, B. W. Lytle, E. H. Blackstone, and N. G. Smedira, "Cardiac valve replacement in patients on dialysis: influence of prosthesis on survival," Annals of Thoracic Surgery, vol. 70, no. 2, pp. 438-441, 2000.

[23] V. Chan, W. R. E. Jamieson, A. G. Fleisher, D. Denmark, F. Chan, and E. Germann, "Valve replacement surgery in endstage renal failure: mechanical prostheses versus bioprostheses," Annals of Thoracic Surgery, vol. 81, no. 3, pp. 857-862, 2006.

[24] K. Tanaka, K. Tajima, Y. Takami et al., "Early and late outcomes of aortic valve replacement in dialysis patients," Annals of Thoracic Surgery, vol. 89, no. 1, pp. 65-70, 2010. 


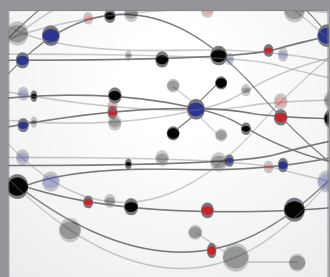

The Scientific World Journal
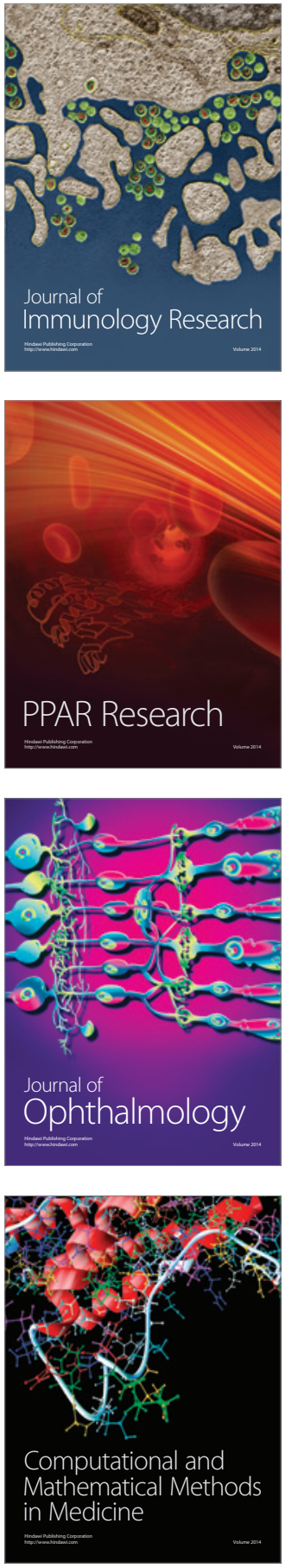

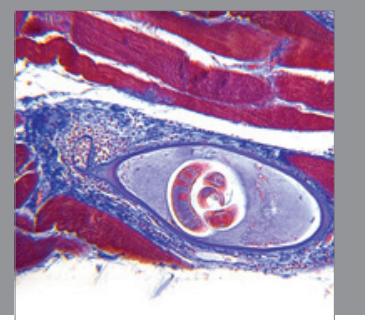

Gastroenterology

Research and Practice
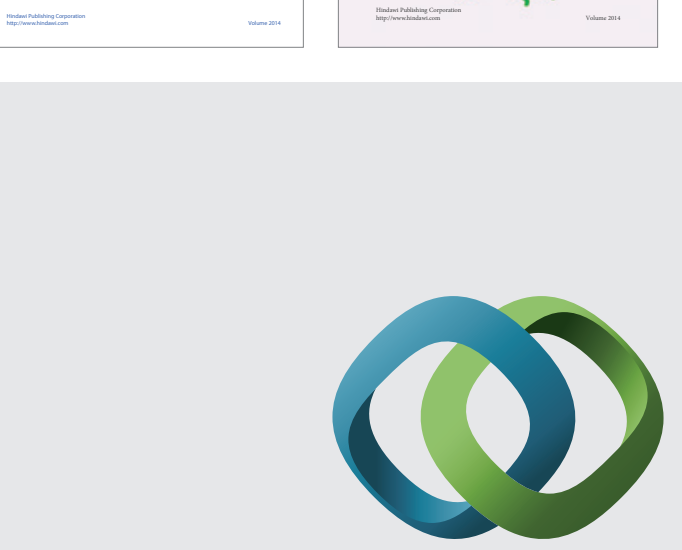

\section{Hindawi}

Submit your manuscripts at

http://www.hindawi.com
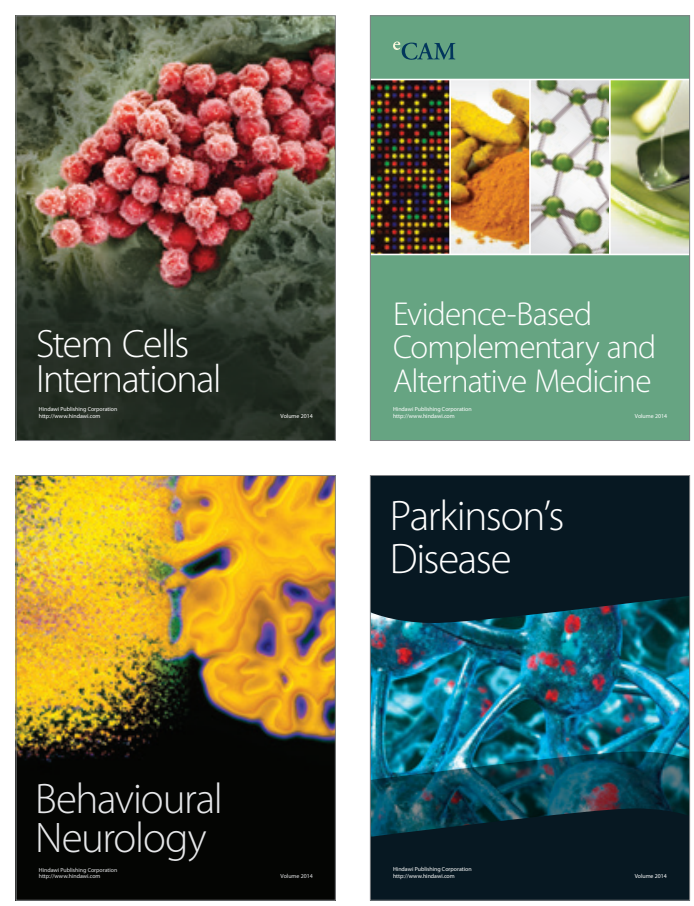

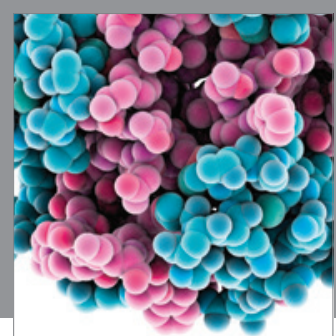

Journal of
Diabetes Research

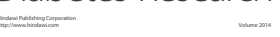

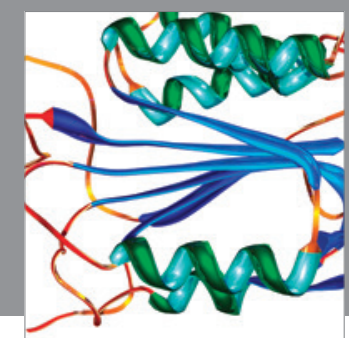

Disease Markers
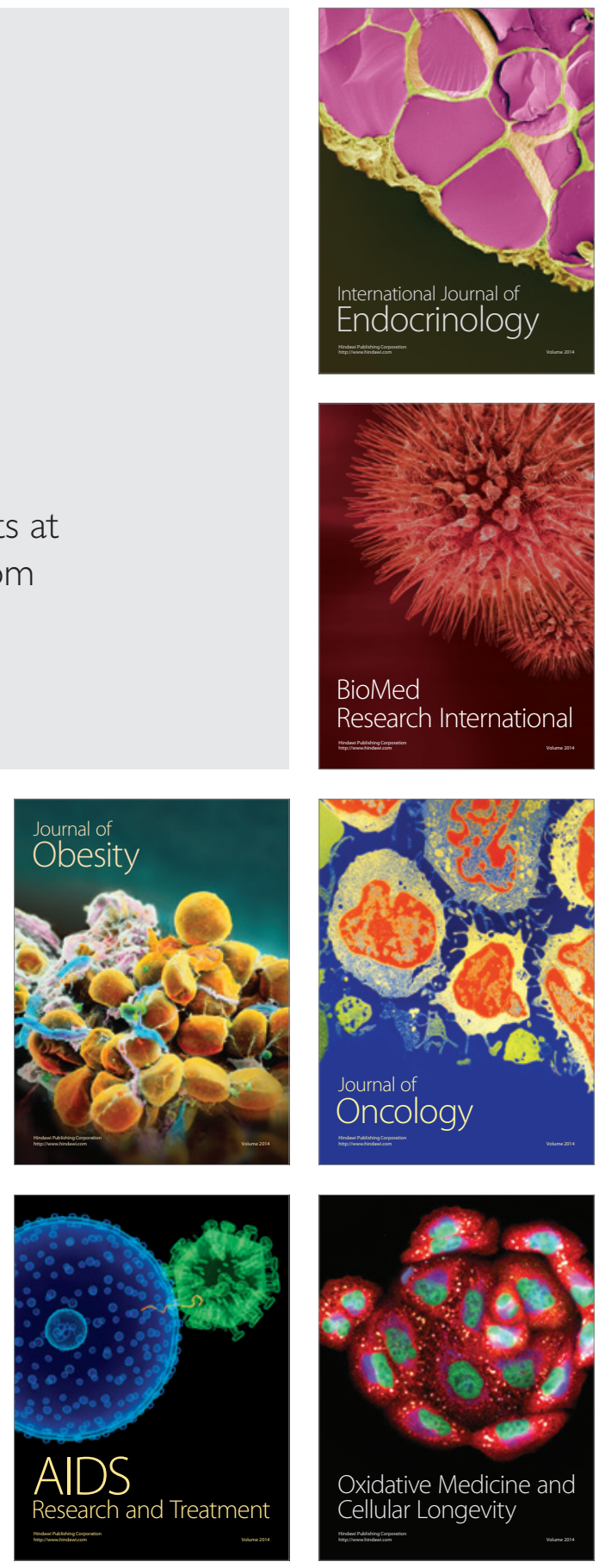